VeritasE Scientia

Vol. 8. N 1.

Enero - Junio del 2019.

ISSN Edición Online: 2617-0639

https://doi.org/10.47796/ves.v8i1.113

\title{
FIBRA SINTÉTICA EN VÍAS A NIVEL DE AFIRMADO Y SU EFECTO EN SUS PROPIEDADES MECÁNICAS, REGIÓN PUNO.
}

\author{
SYNTHETIC FIBER ON ROADS AT THE STATE OF AFFIRMATION AND ITS EFFECT ON ITS MECHANICAL \\ PROPERTIES, PUNO REGION
}

Fausto Ponciano Mamani Mamani ${ }^{1}$

Fausto Alan Lazarte Velarde ${ }^{2}$

Presentado: 20/12/2018

Aceptado: 11/05/2019

Publicado online:17/07/2019

\section{RESUMEN}

La presente investigación surge a través de la observación de los diferentes problemas estructurales que aparecen en los afirmados. Estos deterioros son diversos y muchos de ellos son producto de la mala calidad de los materiales que se emplean en la construcción de las vías afirmadas. Cuyo Objetivo es determinar la repercusión de las propiedades mecánicas de las vías a nivel de afirmado con refuerzo de fibras sintéticas en la provincia de Moho, en el año 2017. Se realizó la comparación de las propiedades mecánicas de afirmados sin adición de fibra sintética en lo que respecta a deformación ( $\mathrm{mm}$ ) $(\bar{X} 1)$, versus las propiedades mecánicas de afirmados con adición de fibras sintéticas con dosificación al $0.1 \%$ del peso de suelo en lo que respecta a deformación $(\mathrm{mm})(\bar{X} 2)$. Para esto se aplicó la correlación $r$ de Pearson y se determinó cómo afecta en el diseño del afirmado. Se usó la correlación $r$ de Pearson. El nivel fue experimental, aplicativa y correlacional. Se requiere de $0.1 \%$ de fibra de polipropileno por peso del afirmado en estado suelto, para mejorar la resistencia al esfuerzo de corte de un camión de 3.44 Tn en una rueda; del mismo modo, producir un aumento del CBR para así reducir el espesor del afirmado inicial en $2 \mathrm{~cm}$.

PALABRAS CLAVE: Fibra Sintética, Suelo, Propiedades mecánicas del suelo, vías, limite líquido, limite plástico, contenido de humedad.

\section{ABSTRACT}

The present investigation arises through the observation of the different structural problems that appear in the affirmed ones. These deteriorations are diverse and many of them are the product of the poor

\footnotetext{
${ }^{1}$ Magíster en Ingeniería Civil con mención en Geotecnia

2 Magíster Académico de la Facultad de Ingeniería de la Universidad Privada de San Carlos, Perú.
} 
quality of the materials used in the construction of the affirmed roads. Objective is to determine the impact of the mechanical properties of the roads at the level of the reinforced synthetic fibers in the province of Moho, in the year 2017. The comparison of the mechanical properties of the synthetic without adding synthetic fiber was carried out. with respect to deformation $(\mathrm{mm})(\bar{X} 1)$, versus the mechanical properties of affirmed with the addition of synthetic fibers with $0.1 \%$ of the weight of soil in terms of deformation (mm) ( $\bar{X} 2$ ). For this, Pearson's r correlation was applied and it was determined how it affects the design of the affirmed. The Pearson $r$ correlation was used. The level was experimental, applicative and correlational. It requires $0.1 \%$ of polypropylene fiber per weight of the loose one in order to improve the resistance to the cutting effort of a truck of 3.44 tons in a wheel; in the same way, produce an increase of the CBR to reduce the thickness of the initial one in $2 \mathrm{~cm}$.

Keywords: Synthetic Fiber, Soil, Mechanical properties of the soil, roads, liquid limit, plastic limit, moisture content.

\section{INTRODUCCIÓN}

En nuestra Región los afirmados se deterioran por fallas como las ondulaciones y ahuellamientos estos se producen por el mezclado pésimo de agregado y ligante que se emplean en la construcción de las vías a nivel de afirmado. Estas fallas en las vías a nivel de afirmado son causadas, por la mala compactación que genera vacíos en el afirmado porque existen fallas plásticas que no son admisibles tanto en la subrasante como en la capa de afirmado. Iniciando los afirmados se ha utilizado fibras naturales que aumenta las propiedades mecánicas del suelo en la construcción. Actualmente se usan las fibras artificiales que se obtienen como excedente de procesos industriales y son susceptibles de ser utilizadas como mejora de diferentes materiales, entre otros el suelo. En el lugar de estudio, los principales deterioros de los afirmados, causan la idea de reforzar al material de afirmado con fibras sintéticas de polipropileno las cuales se encuentran en el mercado y con el paso del tiempo es más usado por los desperdicios que genera la tecnología, estas fibras son más comunes en el mercado. Este refuerzo ha sido planteado con el objetivo de mejorar las propiedades mecánicas de los afirmados, para así poder resistir sin ningún inconveniente cargas importantes de tránsito y quizá lo más importante, tener una vía durable que disminuya el costo de mantenimiento o una reconstrucción posterior. Las vías afirmadas tienen fallas debido al tráfico y el agua, estas fallas presentan deformaciones en la capa de afirmado ya sea por ondulaciones o ahuellamientos, ya que estos son los que causan mayor inseguridad, incomodidad y el costo de mantenimiento para ellos son los más elevados. Las ondulaciones ahuellamientos, baches y otros en los afirmados se presentan por pequeñas deformaciones plásticas cohesivas cada vez que se aplica una carga de un vehículo sobre el afirmado. Afirmados requiere uso de tablas o fórmulas, estas presentan de manera muy conservadora valores de espesores de afirmado considerando para dicho cálculo el CBR de la subrasante y en el tránsito los ejes equivalentes de diseño. Fernández Calvo, 2010 refiere que la mejora de las propiedades geotécnicas por incorporación de fibras textiles sintéticas observada en los ensayos efectuados, es tanto mayor cuanto peor sea el suelo, y cuanto más largas sean las fibras utilizadas. Martínez Santamaría, 2011 concluye que las mejoras importantes parecen relacionarse directamente con la longitud de las fibras. A mayor longitud, mejores propiedades. García Aymar, 2007 en Perú refiere que al aumentar la dosificación de fibra sintética el asentamiento obtenido será menor, independientemente de 
la relación agua - cemento. Aquí, es donde actúa el aditivo Poliheed 770R controlando la trabajabilidad del concreto con fibras sintéticas. El afirmado requiere de un porcentaje de piedra para soportar las cargas, asimismo un porcentaje de arena clasificada según tamaño para llenar los vacíos entre las piedras y dar estabilidad a la capa; y, obligatoriamente un porcentaje de finos plásticos para cohesionar los materiales de la capa de afirmado. Desde la antigüedad se han utilizado fibras naturales para la mejora de las propiedades de los materiales que intervienen en la construcción. En la actualidad se están empezando a emplear fibras artificiales que se obtienen como excedente de procesos industriales y son susceptibles de ser utilizadas como mejora de diferentes materiales, entre otros el suelo. Las fibras pueden ser de origen natural o de origen artificial o sintético. Las fibras naturales de origen animal y vegetal son biodegradables y se usan sólo en los casos que se requiera dicha cualidad. Las fibras naturales minerales (amianto, asbesto) tienen diferentes usos industriales que hoy tienen prohibido su uso por ser cancerígenas. Otras fibras derivadas de metales (alambres) no se usan solas para refuerzo. Hoy por hoy, las fibras sintéticas de mayor producción en el mercado, las más utilizadas y también las de menor coste (posiblemente por ser las que se producen a mayor escala) son PAN, PA 6.6, PA 6, PES, PE, PP. Sus precios no varían mucho unas de otras, son fáciles de encontrar en el mercado.

\section{OBJETIVOS}

Describir los afirmados sin refuerzo de fibra sintética en las propiedades mecánicas de los afirmados en el camino vecinal Jipata - Umuchi del distrito de Moho, región de Puno 2017.

Diseñar la dosificación de fibra sintética óptima en las propiedades mecánicas de los afirmados en el camino vecinal Jipata - Umuchi del distrito de Moho, región de Puno 2017.

\section{METODOLOGÍA}

Investigación exploratoria, descriptiva, explicativa y correlacional. Se realizó la comparación de las propiedades mecánicas de afirmados sin adición de fibra sintética $(\bar{X} 1)$, versus las propiedades mecánicas de afirmados con adición de fibras sintéticas ( $\bar{X}_{2}$ ). Se aplicó coeficiente de correlación $r$ de Pearson de dos muestras. El área de estudio se encuentra en el trayecto de la vía afirmada del camino vecinal Jipata - Umuchi - Moho Km 14+000 al 15+000, de la región de Puno. Con una pendiente aproximada 0 a $12 \%$ en la parte llana, y en las laderas de 12 a $35 \%$ y en las de fuerte inclinación se tiene pendientes superiores a $35 \%$. La población aproximada que se ubica dentro de los dos sectores de estudio es de 4315 habitantes. El tramo de muestra comprende entre el Km 14+000 y 15+000. Para la determinación de las propiedades mecánicas del afirmado reforzado se usaron los siguientes instrumentos: Clasificación de campo y laboratorio (ASTM D2488), Composición granulométrica por mallas (ASTM D422), Contenido de agua natural (ASTM D2216) CASAGRANDE, Límites de consistencia, líquido (ASTM D423) y plástico (ASTM D424), Clasificación de Suelos (ASTM D 2487), Ensayo de Compactación Proctor Modificado (ASTM D 1557), Ensayo de CBR. (ASTM D1883) y Resistencia al corte en compresión no confinada con dos ciclos de carga y descarga (ASTM D2166). Para la ejecución de este ensayo fue necesario tomar 4 muestras de suelo que pasó por el tamiz número 4 para garantizar que solo se le hiciera la prueba al suelo y no hubiera piedras, cada muestra tenía una masa de $3.5 \mathrm{~kg}$ y se organizaron en bolsas a las que se agregaron 100, 200, 300 y $350 \mathrm{ml}$ de agua, luego de esto se procedió a sacar la energía de compactación que arrojó un número de 25 golpes en 3 capas. A cada muestra se le realizó el mismo procedimiento, como resultado se obtuvo el contenido de humedad óptimo de $12.50 \%$ y la densidad máxima seca de $1.920 \mathrm{~g} / \mathrm{cm} 3$ datos con los cuales se desarrollaron los ensayos de corte directo y CBR. Los ensayos de CBR se hacen usualmente en muestras compactadas al contenido de humedad óptima para un suelo específico. Para la elaboración de los ensayos de corte directo se utilizaron 4 clases de muestras diferentes, de los cuales se sacaron los anillos para sus posteriores fallas, estas 
muestras fueron dispuestas de la siguiente manera: una muestra testigo, es decir, sin fibra sintética; otra muestra con una dosificación de fibra de $0.1 \%, 0.5 \%, 1.0 \%$ y por último otra muestra con un porcentaje de $2.00 \%$. Los ensayos de laboratorio fueron aquellos que nos permitieron medir tanto las características físicas como mecánicamente del afirmado reforzado, estos fueron realizados en la Dirección Regional de Transportes y Comunicaciones -Puno. La manera más simple de caracterizar el comportamiento de los afirmados bajo la acción de las cargas producidas por las ruedas de los vehículos es considerando un semi-espacio homogéneo. Un semi- espacio tiene una longitud y un espesor infinito, en el cual las cargas son aplicadas. Para calcular la resistencia del afirmado con o sin refuerzo ante las cargas establecidas, se debe realizar el dibujo de círculos de Mohr, los cuales se trazarán a partir de un estado inicial. Para poder satisfacer las solicitudes de carga a las que se encuentra sometida la capa de afirmado, se realizaron los cálculos de esfuerzos de corte y se compararon con los de resistencia al corte producto de los ensayos de laboratorio. En este cálculo se consideraron dos tipos de carga, una de ellas tomando como modelo el Camión tipo C2 y el segundo considerando el camión de diseño que propone el manual de puentes. Dosificación de fibra de polipropileno $=0.10 \%$ del peso del afirmado suelto. El tránsito es una de las variables más importantes para el análisis de los pavimentos. Para realizar este estudio de tránsito, se contabilizaron los vehículos durante siete días seguidos en el mes de agosto, clasificándolos por sentido, hora y tipo.

\section{RESULTADOS}

Las características de CBR de las capas superficiales de Afirmado Existente, que conforman la plataforma y el terreno natural como subrasante registra las siguientes características de soporte:

Tabla 1

Módulo resilente (kpsi) y cbr (\%) de la subrasante

\begin{tabular}{llcc}
\hline Sector & RED VIAL 01 & Mr & CBR \\
\cline { 3 - 4 } & & Mpsi & $\%$ \\
\hline 1 & Moho - Umuchi 7.00 & 16.5 & \\
\hline 2 & Umuchi - Jipata 15.00 & 14.02 & 22.9 \\
\hline
\end{tabular}

El análisis granulométrico se realizó mediante la norma MTC E 107-2000 ASTM D 422 a través de tamizado. El material que se utilizó proveniente de la cantera de M (km 11+000 del camino Umuchi Moho), como demuestra la curva posee un desfase al comienzo debido a la presencia de piedra de $2^{\prime \prime}$ a $2.5^{\prime \prime}$. (Fig .1)

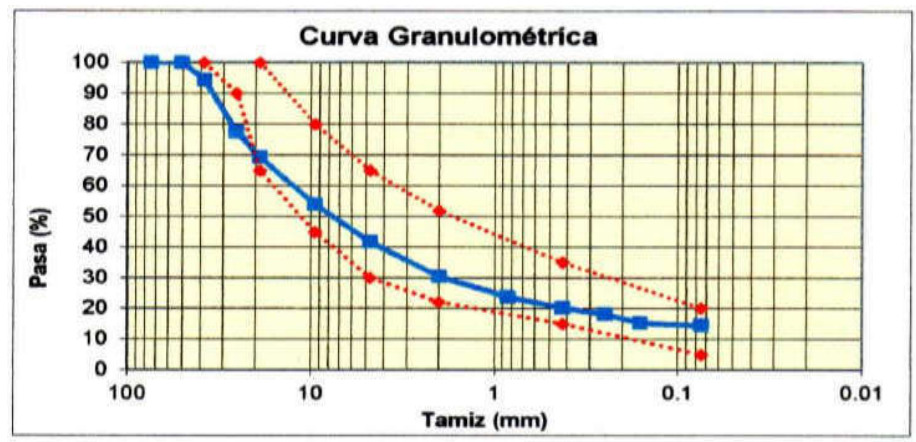

Figura 1. Curva granulométrica 
El límite líquido fue realizado mediante la norma ASTM D 423, y el límite plástico se realizó mediante la norma ASTM D 424. (Fig. 2 y Tabla 2). El ensayo de Proctor modificado del material de afirmado fue realizado mediante la norma MTC E 115 - 2000, ASTM D 1557. (Fig.3 y Tabla 3)

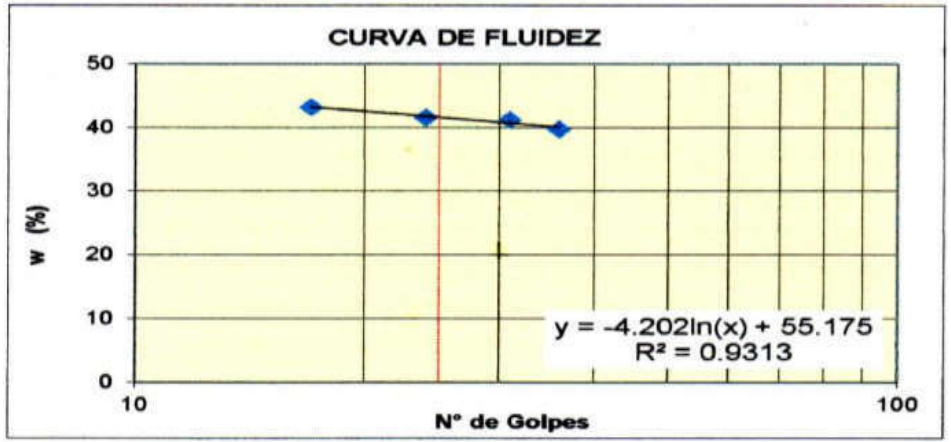

Figura 2. Límites de consistencia

Tabla 2

Resultados Límites de Consistencia

\begin{tabular}{lc}
\hline Límite líquido & 41. \\
Límite & $2 \overline{9}$ \\
Plóction de & 97 \\
Índice de & 11. \\
Plasticidad & 68 \\
\hline
\end{tabular}

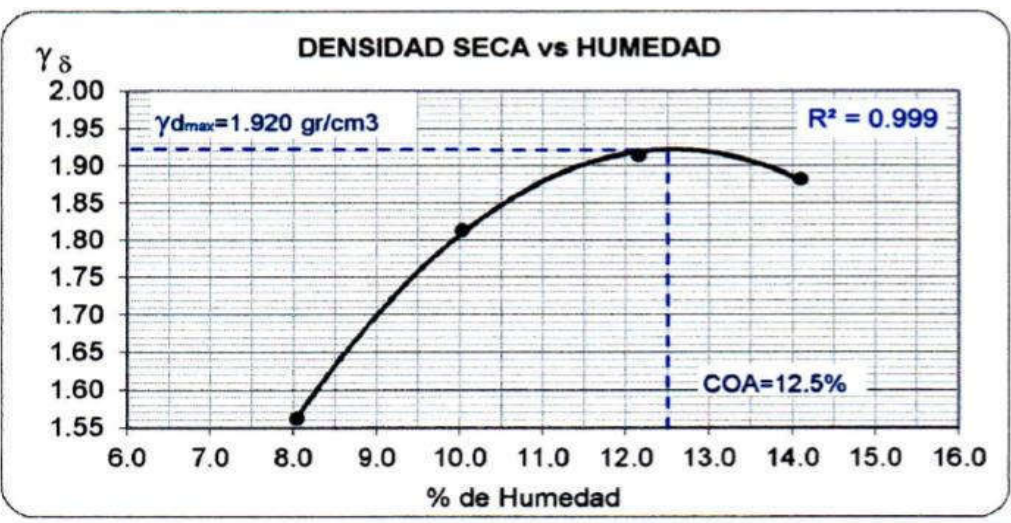

Figura 3. Curva de compactación

Tabla 3

Resultados Proctor Modificado

\begin{tabular}{lc}
\hline Densidad máxima seca & $1.920 \mathrm{gr} / \mathrm{cm}^{3}$ \\
Óptimo \% de humedad & $12.50 \%$ \\
\hline
\end{tabular}

La fibra sintética para el refuerzo de afirmados utilizando la dosificación óptima de fibra sintética al 0.10\% en el camino vecinal Jipata, mejora las propiedades mecánicas, debido al aumento de la resistencia al corte y al incremento del CBR (Tabla 4) 


\section{Tabla 4}

\section{Propiedades mecánicas del Ensayo de Corte Directo}

\begin{tabular}{|c|c|c|c|c|c|c|}
\hline \multirow[b]{3}{*}{ ENSAYO DE CORTE DIRECTO } & \multicolumn{6}{|c|}{ PROPIEDADES MECÁNICAS } \\
\hline & \multirow{2}{*}{$\begin{array}{l}\text { Cohesión } \\
\left(\mathrm{Kg} / \mathrm{cm}^{2}\right)\end{array}$} & \multirow{2}{*}{$\begin{array}{l}\text { Ángulo de } \\
\text { fricción } \\
\text { (grados) }\end{array}$} & \multicolumn{2}{|c|}{ CAMIÓN C2 } & \multicolumn{2}{|c|}{ CAMIÓN DE DISEÑO } \\
\hline & & & $\begin{array}{l}\text { Esfuerzo } \\
\text { normal } \\
\left(\mathrm{Kg} / \mathrm{cm}^{2}\right)\end{array}$ & $\begin{array}{l}\text { Resistencia } \\
\text { al corte } \\
\left(\mathrm{Kg} / \mathrm{cm}^{2}\right)\end{array}$ & $\begin{array}{l}\text { Esfuerzo } \\
\text { normal } \\
\left(\mathrm{Kg} / \mathrm{cm}^{2}\right)\end{array}$ & $\begin{array}{l}\text { Resistencia } \\
\text { al corte } \\
\left(\mathrm{Kg} / \mathrm{cm}^{2}\right)\end{array}$ \\
\hline Afirmado sin adición de fibra sintética & 0.55 & $24.78^{\circ}$ & 1.794 & 1.96 & 2.616 & 2.51 \\
\hline Afirmado con dosificación de fibra sintética a $0.1 \%$ del peso del suelo & 0.42 & $32.76^{\circ}$ & 1.794 & 2.43 & 2.616 & 3.24 \\
\hline $\begin{array}{l}\text { Porcentaje de incidencia del afirmado sin fibra sintética versus el } \\
\text { afirmado con } 0.10 \% \text { de doisificación óptima de fibra sintética }\end{array}$ & $-23.64 \%$ & $32.20 \%$ & - & $23.98 \%$ & - & $29.08 \%$ \\
\hline
\end{tabular}

En el caso del corte directo se realizó un análisis estático de las cargas actuantes, para las cuales es necesaria la utilización de $0.1 \%$ de fibra, esto significa un incremento en el ángulo de fricción de $32.20 \%$, la cohesión se reduce a 23.64\%, la resistencia al corte incrementa en el Camión C2 en 23.98\% y para un camión de diseño de $29.08 \%$. Estas propiedades repercuten de la durabilidad del afirmado al aumentar la resistencia al corte. (Tabla 5)

\section{Tabla 5}

Propiedades mecánicas del ensayo de California Bearing Ratio (CBR)

\begin{tabular}{lllll}
\hline CALIFORNIA BEARING RATIO (CBR) & CBR (\%) & Mr (PSI) & $\begin{array}{l}\text { Número } \\
\text { estructural a3 }\end{array}$ & $\begin{array}{l}\text { Espesor } \\
\text { (cm) }\end{array}$ \\
\hline $\begin{array}{l}\text { Afirmado sin adición de fibra sintética } \\
\begin{array}{l}\text { Afirmado con dosificación de fibra sintética a 0.1\% del peso del } \\
\text { suelo }\end{array}\end{array}$ & 54.03 & 17199.09 & 0.11655439 & 20.00 \\
$\begin{array}{l}\text { Porcentaje de incidencia del afirmado si fibra sintética versus el } \\
\text { afirmado con 0.10\% de doisificación óptima de fibra sintética }\end{array}$ & $35.08 \%$ & $8.03 \%$ & 6.12416822 & 18.00 \\
\hline
\end{tabular}

En el caso del CBR, se reduce el espesor del afirmado en función al incremento del CBR producto de la añadidura de fibra, siendo la máxima reducción de espesor $2 \mathrm{~cm}$, lo cual significaría un ahorro del 10\% de afirmado. La dosificación que nos permite disminuir el espesor es de $0.10 \%$ de fibra sintética.

Las deformaciones de los afirmados sin refuerzo de fibra sintética tienen correlación respecto a la deformación del afirmado con dosificación óptima de fibra sintética a $0.10 \%$; La correlación estadística mediante $r$ de Pearson confirma que existe correlación de las deformaciones producidas por la fuerza tangencial en un área de $506.71 \mathrm{~cm} 2$ esto debido a las cargas normales constantes en los ensayos de $w n=17.71, w n=31.40$ y 45.50 .

\section{Tabla 6}

Propiedades mecánicas del Ensayo de Corte Directo

\begin{tabular}{|c|c|c|c|c|c|c|}
\hline \multirow[b]{3}{*}{ ENSAYO DE CORTE DIRECTO } & \multicolumn{6}{|c|}{ PROPIEDADES MECÁNICAS } \\
\hline & \multirow{2}{*}{$\begin{array}{l}\text { Cohesión } \\
\left(\mathrm{Kg} / \mathrm{cm}^{2}\right)\end{array}$} & \multirow{2}{*}{$\begin{array}{l}\text { Ángulo de } \\
\text { fricción } \\
\text { (grados) }\end{array}$} & \multicolumn{2}{|c|}{ CAMIÓN C2 } & \multicolumn{2}{|c|}{ CAMIÓN DE DISEÑO } \\
\hline & & & $\begin{array}{l}\text { Esfuerzo } \\
\text { normal } \\
\left(\mathrm{Kg} / \mathrm{cm}^{2}\right)\end{array}$ & $\begin{array}{l}\text { Resistencia } \\
\text { al corte } \\
\left(\mathrm{Kg} / \mathrm{cm}^{2}\right)\end{array}$ & $\begin{array}{l}\text { Esfuerzo } \\
\text { normal } \\
\left(\mathrm{Kg} / \mathrm{cm}^{2}\right)\end{array}$ & $\begin{array}{l}\text { Resistencia } \\
\text { al corte } \\
\left(\mathrm{Kg} / \mathrm{cm}^{2}\right)\end{array}$ \\
\hline Afirmado sin adición de fibra sintética & 0.55 & $24.78^{\circ}$ & 1.794 & 1.96 & 2.616 & 2.51 \\
\hline Afirmado con dosificación de fibra sintética a $0.1 \%$ del peso del suelo & 0.42 & $32.76^{\circ}$ & 1.794 & 2.43 & 2.616 & 3.24 \\
\hline Afirmado con dosificación de fibra sintética a $0.5 \%$ del peso del suelo & 0.44 & $38.92^{\circ}$ & 1.794 & 3.05 & 2.616 & 4.08 \\
\hline
\end{tabular}


Tabla 7

Propiedades mecánicas del ensayo de California Bearing Ratio (CBR)

\begin{tabular}{|c|c|c|c|c|}
\hline CALIFORNIA BEARING RATIO (CBR) & CBR (\%) & $\mathrm{Mr}$ (PSI) & $\begin{array}{l}\text { Número } \\
\text { estructural a3 }\end{array}$ & $\begin{array}{l}\text { Espesor } \\
(\mathrm{cm})\end{array}$ \\
\hline Afirmado sin adición de fibra sintética & 40 & 16199.09 & 0.11655439 & 20.00 \\
\hline Afirmado con dosificación de fibra sintética a $0.1 \%$ del peso del suelo & 54.03 & 17499.75 & 0.12416822 & 18.00 \\
\hline Afirmado con dosificación de fibra sintética a $0.5 \%$ del peso del suelo & 58.9 & 17873.09 & 0.12624931 & 18.00 \\
\hline Afirmado con dosificación de fibra sintética a 1.0\% del peso del suelo & 64.23 & 18247.85 & 0.12829504 & 18.00 \\
\hline Afirmado con dosificación de fibra sintética a $2.0 \%$ del peso del suelo & 10.68 & 10486.58 & 0.07368390 & 31.00 \\
\hline
\end{tabular}

La inclusión de fibra sintética con dosificación de $0.10 \%$. es óptima para el diseño de afirmados porque aumenta la resistencia al corte en diferencia a la no inclusión de fibra sintética como se muestra en la tabla 6.

El ensayo de corte directo se obtuvo un aumento del ángulo de fricción entre 32\% y un 73\% para una dosificación del $0.10 \%$ al $1.0 \%$ de fibra, sin embargo, para el $2.0 \%$ de fibra el ángulo de fricción solamente llego a un aumento del $33 \%$. Esto porque la fibra sintética se une a las partículas pétreas y mejora el ángulo de fricción. Se obtuvo la disminución de la cohesión, esto debido a que la cohesión se produce por enlaces electroquímicos, los cuales se rompen en la medida que son cargados positivamente por las fibras de polipropileno. La disminución de la cohesión vario entre 20\% al 76\%. (Tabla 7).

El ensayo de CBR, produjo incrementos; para $0.10 \%$ de dosificación de fibra, en $14.03 \%$; para 0.5\% de dosificación, en $18.90 \%$; para $1.0 \%$ de dosificación en $24.23 \%$; pero para la dosificación de $2.0 \%$ el CBR disminuyo en $29.32 \%$, todos con respecto al afirmado sin fibra sintética. La dosificación mayor a $1.0 \%$ no es recomendable, ya que las características mecánicas se ven afectadas, esto producto al acolchonamiento que no permite una adecuada homogenización, haciendo que las partículas entren en suficiente contacto.

La capa de afirmado reforzada con fibra sintética, necesitó el 0.10\% de fibra para resistir el esfuerzo de corte que produce una rueda de $5.438 \mathrm{Tn}$, esto incrementa el CBR del afirmado y provocar una disminución de $2 \mathrm{~cm}$ al espesor de $20 \mathrm{~cm}$. siendo $18 \mathrm{~cm}$. el nuevo espesor del afirmado

\section{DISCUSIÓN}

Se recomienda el uso de una mezcladora de concreto para realizar esta actividad. El proceso de mezclado es la ruta crítica en el proceso constructivo, por lo que se podría recomendar el uso de una maquinaria especializada para este trabajo, usar una mezcladora de suelos o quizá una recicladora en campo lo cual evitaría que las fibras puedan quedar sueltas y ser transportadas por el aire a las áreas próximas a la carretera. Sería importante realizar este trabajo en otras partes de la estructura de la carretera, para estabilizar y reforzar, caso de bases, sub-bases, suelos de fundación, capa anticontaminante, etc. Así mismo se puede variar las longitudes de fibra, tipo de fibra y las marcas. También se hace necesario realizar tramos experimentales in situ con un control a base de ensayos a mayor escala que los de laboratorio, con la finalidad de ver el comportamiento mecánico de la fibra sintética en campo y determinar las dosificaciones de fibra sintética respecto al tipo de suelo encontrado.

\section{REFERENCIAS BIBLIOGRÁFICAS}

Asociación Técnica de Carreteras Comité de Geotecnia Vial. Refuerzo de suelos con fibra sintética: Perú; 2013

Badillo E. Mecánica de suelos. México: Editorial Limusa; 1976.

Beltrán M L. VIII Congreso Panamericano de Mecánica de Suelos e Ingeniería de Fundaciones =. [Bogotá]: Sección de Publicaciones, Universidad Nacional de Colombia; 1987.

Berry Preid D. Mecánica de suelo. Santa Fé de Bogotá: McGraw-Hill; 1993. 
Céspedes Abanto, José María. Los pavimentos en las vías terrestres: Calles, carreteras y aeropistas. Cajamarca: Universidad Nacional de Cajamarca; 2016.

Coronado García O. Construcción de una máquina de corte directo para estudio de suelos reforzados con geosintéticos. Santa Fé de Bogotá: Uniandes; 1995.

Das B. Principles of geotechnical engineering. Boston: PWS; 1985.

Devore J. Sánchez Fragoso F. Probabilidad y estadística para ingeniería y ciencias. México, D.F.: Thomson; 2005.

Dirección General de Caminos y Ferrocarriles M. Manual para el diseño de caminos no pavimentados de bajo volumen de tránsito. Tarea Asociación Gráfica Educativa; 2008.

Dirección Regional de Transportes y Comunicaciones -Puno- Laboratorio de geotecnia de mecánica de suelos, concreto, pavimentos y ensayos de materiales. Gobierno Regional de Puno; 2016.

Eyssautier de la Mora M. Metodología de la investigación. México: International Thomson; 2006.

García Avilés A. Introducción a la metodología de investigación científica. México: Plaza y Valdés; 1997.

Fernández Calvo C. Estudio experimental de refuerzo de suelos con fibras sintéticas. Casilla: Geogaceta; 2006.

Fernández Calvo C, Martínez Santa María J, Thode Mayoral J. Refuerzo de suelos con fibra sintética. Andalucía: asociación técnica de carreteras; 2010.

Hernández R, Fernández C, Baptista P. Metodología de la investigación. 4th ed. Bogotá: McGrawHill; 2006. Jiménez Salas J, Justo Alpañes J, Serrano González A. Geotecnia y cimientos. Madrid: Rueda; 1975.

Lambe TWhitman R. Mecánica de suelos. México: Editorial Limusa; 1972.

Rico Rodríguez A. La ingeniería de suelos en las vías terrestres. México D.F: Limusa; 1977.

Terzaghi K, Peck R, Moretto O. Mecánica de suelos en la ingeniería práctica. Barcelona: El Ateneo Editorial; 1963.

Triola M Pineda Ayala M. Probabilidad y estadística. México: Pearson Educación; 2004.

Yang H. Huang. Pavement Analysis and Design. U.S: Prentice Hall; 2003. 